\title{
Identification of a lactate-quinone oxidoreductase in Staphylococcus aureus that is essential for virulence
}

\author{
James R. Fuller ${ }^{\dagger}$, Nicholas P. Vitko ${ }^{\dagger}$, Ellen F. Perkowski, Eric Scott, Dal Khatri, Jeffrey S. Spontak, \\ Lance R. Thurlow and Anthony R. Richardson*
}

Department of Microbiology and Immunology, University of North Carolina at Chapel Hill, Chapel Hill, NC, USA

Edited by:

David Heinrichs, University of

Western Ontario, Canada

\section{Reviewed by:}

Alain Charbit, University Paris

Descartes, INSERM U1002, France

Paul Douglas Fey, University of

Nebraska Medical Center, USA

Susanne Engelmann,

Ernst-Moritz-Arndt University,

Germany

\section{*Correspondence:}

Anthony R. Richardson, Department of Microbiology and Immunology, University of North Carolina at Chapel Hill, 116 Manning Dr, CB7290, Chapel Hill, NC 27599, USA.

e-mail: anthony_richardson@

med.unc.edu

${ }^{\dagger}$ James R. Fuller and Nicholas P. Vitko have contributed equally to this work and are designated as co-authors.
Staphylococcus aureus is an important human pathogen commonly infecting nearly every host tissue. The ability of $S$. aureus to resist innate immunity is critical to its success as a pathogen, including its propensity to grow in the presence of host nitric oxide (NO.). Upon exogenous NO. exposure, S. aureus immediately excretes copious amounts of L-lactate to maintain redox balance. However, after prolonged NO--exposure, S. aureus reassimilates L-lactate specifically and in this work, we identify the enzyme responsible for this L-lactate-consumption as a L-lactate-quinone oxidoreductase (Lqo, SACOL2623). Originally annotated as Mqo2 and thought to oxidize malate, we show that this enzyme exhibits no affinity for malate but reacts specifically with L-lactate $\left(K_{\mathrm{M}}=\sim 330 \mu \mathrm{M}\right)$. In addition to its requirement for reassimilation of L-lactate during NO--stress, Lqo is also critical to respiratory growth on L-lactate as a sole carbon source. Moreover, $\Delta / q o$ mutants exhibit attenuation in a murine model of sepsis, particularly in their ability to cause myocarditis. Interestingly, this cardiac-specific attenuation is completely abrogated in mice unable to synthesize inflammatory NO. (iNOS ${ }^{-l-}$ ). We demonstrate that $S$. aureus NO--resistance is highly dependent on the availability of a glycolytic carbon sources. However, S. aureus can utilize the combination of peptides and L-lactate as carbon sources during NO--stress in an Lqo-dependent fashion. Murine cardiac tissue has markedly high levels of L-lactate in comparison to renal or hepatic tissue consistent with the NO--dependent requirement for Lqo in S. aureus myocarditis. Thus, Lqo provides $S$. aureus with yet another means of replicating in the presence of host NO.

Keywords: Staphylococcus aureus, lactate-quinone oxidoreductase, virulence, myocarditis, pericarditis, metabolism

\section{INTRODUCTION}

Staphylococcus aureus is a pathogen of tremendous importance to human health (Diekema et al., 2001). The Gram-positive bacterium can be commonly isolated from nasal passages, axillae, and perinea of healthy carriers (Mermel et al., 2011). While the most common disease presentations of $S$. aureus are skin/soft tissue infections, it is also a frequent cause of sepsis, endocarditis, and osteomyelitis (Klevens et al., 2007). The latter conditions can be complicated by bacterial spread to cardiac muscle resulting in purulent abscesses or myocarditis (Wasi and Shuter, 2003). S. aureus can also seed the pericardial space resulting in pericarditis necessitating surgical drainage followed by extensive intravenous antibiotic administration (Klacsmann et al., 1977; Mookadam et al., 2009). While pericarditis and myocarditis are not the most frequent disease presentations associated with $S$. aureus infections, this organism is the one of the most common causes of both life-threatening conditions (Wasi and Shuter, 2003; Mookadam et al., 2009). It is thought that myocarditis and pericarditis are rare complications of sepsis because bacteria in general do not efficiently colonize muscle tissue. Indeed, bacterial infections in skeletal muscles (pyomyositis/myositis) are even more rare than infections of cardiac muscle tissue. However, as with pericarditis and myocarditis, the most common cause of bacterial myositis and pyomyositis is S. aureus (Pannaraj et al., 2006). Thus, this pathogen has a propensity to colonize and thrive in nearly every sterile site in the body, even environments not conducive to supporting most other bacterial pathogens.

In order for $S$. aureus to inhabit sterile sites within the human body, it must be able to resist numerous host innate immune effectors (Foster, 2005). Nitric oxide (NO.) is a key broad-spectrum antimicrobial host effector that is essential for the efficient clearance of a variety of microbial pathogens (DeGroote and Fang, 1999). As with most other immune effectors, S. aureus is uncommonly resistant to the effects of exogenous NO. (Richardson et al., 2006, 2008; Hochgrafe et al., 2008). This NO--resistance results from the induction of a metabolic state that circumvents the effects of this cytotoxic radical (Richardson et al., 2008). NO. is known to interfere with various bacterial metabolic pathways including the tricarboxylic acid cycle, aerobic respiration, fatty acid metabolism, pyruvate metabolism, and nucleic acid synthesis (Richardson et al., 2008, 2009, 2011). While we do not fully understand how $S$. aureus accommodates the loss of multiple NO--sensitive targets, progress has been made with regard to $S$. aureus adaptation to the redox imbalance associated with NO--stress. Upon exposure to exogenous NO , S. aureus ceases to respire and shifts into a fermentative metabolic state concomitant with increased excretion of L-lactate 
(Richardson et al., 2008). The reduction of pyruvate to L-lactate provides redox balance by regenerating $\mathrm{NAD}^{+}$in a cell unable to utilize the respiration-dependent NADH-dehydrogenase (Complex I of the electron transport chain). In S. aureus most of the L-lactate production is catalyzed by lactate dehydrogenase 1 (Ldh1), an allele that is unique to $S$. aureus and not found in other staphylococci (Richardson et al., 2008). Coincidentally, other staphylococcal species are incapable of robust growth during NO-stress (Richardson et al., 2008). S. aureus additionally possesses another L-lactate dehydrogenase (Ldh2) that is shared among most other staphylococcal species. However, Ldh2 is expressed even in the absence of NO. and, given the strong induction of $l d h 1$ following NO--exposure, Ldh2 offers only a minor contribution to redox balance during NO--stress (Richardson et al., 2008). Finally, S. aureus encodes a D-lactate dehydrogenase (Ddh) that also produces D-lactate during NO--exposure, albeit to a much lower level than the L-enantiomer. Thus, $S$. aureus possesses three lactatedehydrogenases all of which regenerate $\mathrm{NAD}^{+}$with Ldh1 playing the major redox-balancing role during growth in the presence of exogenous NO.

However, a series of elegant studies conducted nearly a halfcentury ago characterized NAD-independent L-lactate dehydrogenase ( $i \mathrm{LDH}$ ) activity in S. aureus (Stockland and San Clemente, 1969). iLDHs oxidize L-lactate to pyruvate with a concomitant reduction of the respiratory quinone pool (Stevenson and Holdsworth, 1973). Electrons are eventually donated to terminal acceptors, and in $S$. aureus L-lactate oxidation was shown to be coupled to the reduction of ferric iron, oxygen, or nitrate (Theodore and Weinbach, 1974; Lascelles and Burke, 1978; Tynecka and Malm, 1995). While many iLDH enzymes exist in the microbial world, no such gene is annotated in any of the current S. aureus genome sequences (more than 14 to date; Stevenson and Holdsworth, 1973). Moreover, homology searches using known $i$ LDH sequences were unsuccessful at identifying a gene encoding such an enzyme in $S$. aureus. Thus, the identification of a putative fourth lactate utilization gene responsible for observed enzymatic activity has proved elusive.

Here we identify the gene encoding the $S$. aureus $i \mathrm{LDH}$ as SACOL2623 originally annotated as Mqo2, a malate-quinone oxidoreductase. Given that malate is chemically similar to L-lactate and that malate- and L-lactate-dependent enzymes are phylogenetically related, the coding genes are occasionally misannotated (Madern, 2002). We show that purified Mqo2 has no affinity for malate and thus we suggest the protein be renamed Lqo. We characterize the contribution of Lqo to S. aureus catabolism of L-lactate both in the presence and absence of exogenous NO. Lqo orthologs are present, within similar genetic environments, in the sequenced genomes of all Staphylococcus species. We also define a role for Lqo in $S$. aureus virulence in a murine model of sepsis. Specifically $\Delta$ lqo mutants are defective for growth within murine cardiac tissue in the presence of host NO. We show that while S. aureus NO--resistance is predicated on the presence of a glycolytic carbon source, specifically combining L-lactate with peptides also provides $S$. aureus with the means to replicate in the presence of NO. in an Lqo-dependent manner. The high levels of both L-lactate and protein in murine cardiac tissue are consistent with the specific NO--dependent requirement for Lqo in murine septic myocarditis/pericarditis. Thus, Lqo represents a key metabolic enzyme allowing $S$. aureus to utilize elevated host L-lactate associated with active muscle tissue and, at the same time, resist the cytostatic effects of host NO.

\section{RESULTS}

\section{STAPHYLOCOCCUS AUREUS HARBORS AN UNIDENTIFIED L-LACTATE CATABOLIC ENZYME}

Upon exposure to exogenous NO., S. aureus evokes fermentative metabolism to contend with the propensity of NO to block respiration. Accordingly, L-lactate, and to a lesser extent, D-lactate are both produced from pyruvate providing the cell with redox balance (Figures 1A,B). However, after prolonged exposure to $\mathrm{NO}$, L-lactate is specifically reassimilated but $\mathrm{D}$-lactate is not (Figure 1B). It should be noted that this L-lactate-consumption occurs despite the presence of excess glucose (data not shown). We reasoned that reversal of the NAD-dependent Ldh1, Ldh2, or Ddh reactions was not responsible for the observed L-lactateconsumption, because: 1 . the reverse reaction would be detrimental to the redox state of the cell and 2. both L-lactate and D-lactate would have been consumed since both reactions are reversible, enzymes for both L-lactate and D-lactate are highly expressed during NO--stress (Richardson et al., 2006) and both enantiomers can support growth of WT S. aureus. Indeed, Ddh allows for Dlactate production anaerobically as well as its aerobic utilization (Figure 1C). Moreover, the $\Delta d d h$ mutant can neither produce nor consume D-lactate (Figure 1C). In contrast, while mutants lacking Ldh1 and Ldh2 do not produce L-lactate anaerobically, the $\Delta l d h 1 \Delta l d h 2$ mutant still grows aerobically on L-lactate as a sole carbon source (Figure 1C). This is not due to racemase activity converting L-lactate to D-lactate as the triple $\Delta l d h 1 \Delta l d h 2 \Delta d d h$ mutant still grows on L-lactate aerobically (data not shown). Thus, $S$. aureus must encode another L-lactate catabolic enzyme that is irreversible (no L-lactate production in the $\Delta l d h 1 \Delta l d h 2$ mutant) and likely does not use $\mathrm{NAD}^{+}$as a cofactor as this would represent a futile cycle with the high Ldh1 levels in NO--exposed cells. The enzyme responsible for the previously described iLDH activity could explain these observations.

\section{SACOL2623 (mqo2) ENCODES THE $S$. AUREUS iLDH ACTIVITY}

No gene in any sequenced $S$. aureus genome is predicted to encode a putative $i \mathrm{LDH}$, nor are there any identifiable homologs of wellcharacterized $i \mathrm{LDH}$ genes in S. aureus. However, S. aureus does encode two malate-quinone oxidoreductases (Mqo1 and Mqo2) that are thought to participate in the TCA cycle since this organism lacks an NAD-dependent malate dehydrogenase (Figure 2A). These enzymes are predicted to use an FAD-cofactor to assist in transferring electrons directly to the respiratory quinone pool, and therefore would oxidize their substrates independently of $\mathrm{NAD}^{+}$. Since malate and lactate are structurally similar in that malate is essentially a carboxylated lactate (Figure 2A), we reasoned that one or both of the annotated Mqo enzymes may react with L-lactate and encode the $S$. aureus $i \mathrm{LDH}$. Indeed, combining a deletion of mqo2, but not mqo1, with the $\Delta l d h 1 \Delta l d h 2$ mutation abolished the ability to grow aerobically on L-lactate as a sole carbon source (Figure 2B). Thus, despite the juxtaposition of mqo1 to a gene encoding a predicted L-lactate permease (Figure $2 \mathbf{A}$ ), it 


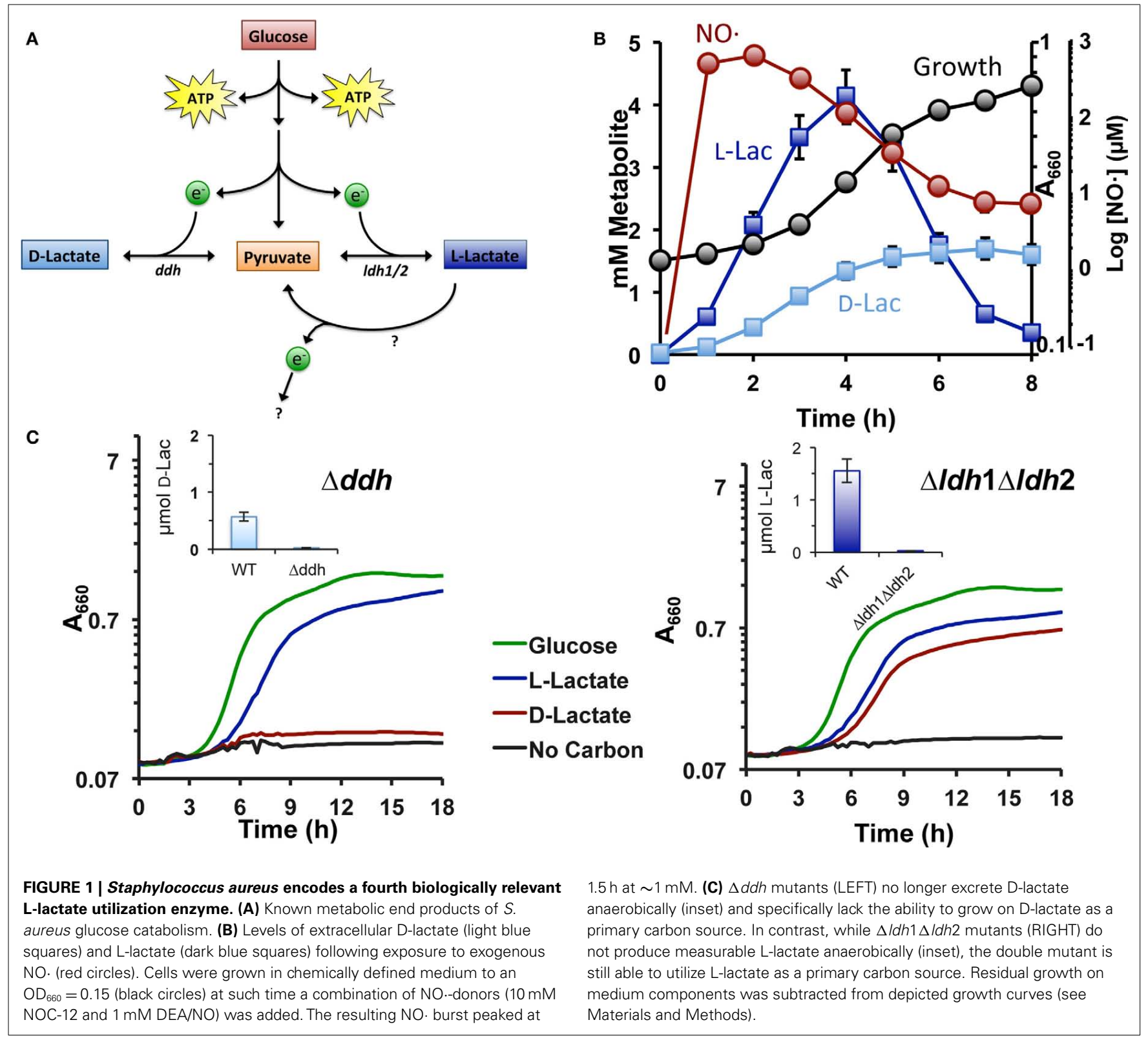

appears that Mqo2 reacts with L-lactate (Figure 2B). Additionally, a $\Delta m q o 2$ mutant, but not $\Delta m q o 1$, was unable to reassimilate L-lactate during prolonged NO-exposure (Figure 2C). However, the lack of L-lactate-reassimilation in the $\Delta m q o 2$ mutant had little reproducible effect on growth in the presence of NO. when glucose was used as the primary carbon source, and there were no effects on the rate of glucose consumption nor glucose yield (mg biomass/mg glucose consumed, data not shown). These data suggest that Mqo2 can oxidize L-lactate to pyruvate and therefore comprises the $S$. aureus iLDH activity. However, while S. aureus $i \mathrm{LDH}$ is active in the presence of NO. (Figure 1B), its role during growth on glucose under NO--stress is still unclear.

In order to distinguish between Mqo2 having dual specificities and the possibility that it only has affinity for L-lactate, we performed enzymatic assays on membrane fractions from single mutant strains to determine substrate specificity. The $\Delta m q o 1$ mutant (only expressing Mqo2) exhibited L-lactate-specific activity but no real activity with malate (Figure 3). Conversely, a $\Delta m q o 2$ mutant membrane preparation reacted readily with malate, but not L-lactate (Figure 3). Membrane preparations from neither the $\Delta m q o 1$ nor the $\Delta m q o 2$ mutant reacted with D-lactate or pyruvate suggesting that Mqo2 was specific for Llactate and the enzyme was non-reversible (data not shown). Finally, His ${ }_{6}$-tagged versions of both enzymes were purified and affinities for malate and L-lactate were determined in an in vitro enzyme assay. While Mqo2 had significant affinity for L-lactate $\left(K_{\mathrm{M}}=\sim 300 \mu \mathrm{M}\right.$, Figure 3), it did not react readily with malate. Again, Mqo1 had high affinity for malate but no detectible reaction with L-lactate (Figure 3). Since Mqo2 does not exhibit any substrate specificity for malate and efficiently oxidizes 
A

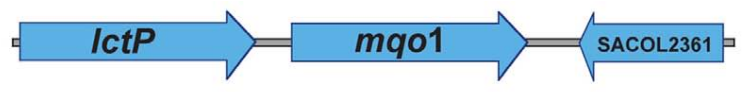

SACOL2624
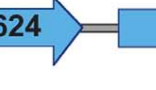

$m q 02$

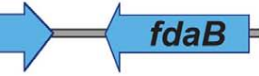

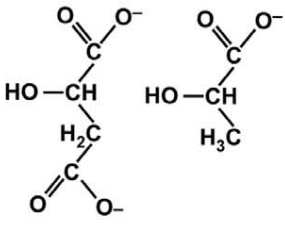

S-malate L-lactate

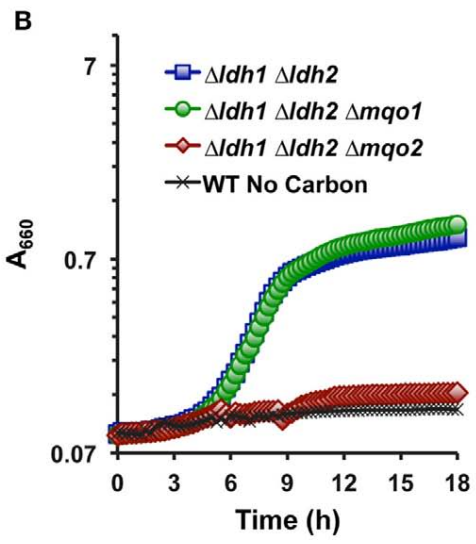

FIGURE 2 | Mqo2 is responsible for the elusive $S$. aureus iLDH activity. (A) Genetic organization of the two annotated malate-quinone oxidoreductase genes. mqo1 is between an L-lactate permease and a hypothetical gene, while $m q 02$ is found between a putative acetyl-CoA synthase (acs, SACOL2624) and a class I fructose 1,6-bisphosphate aldolase, fdaB. (B) Specifically combining a $\Delta m q 02$ mutation to the $\Delta / d h 1 \Delta / d h 2$ background

C

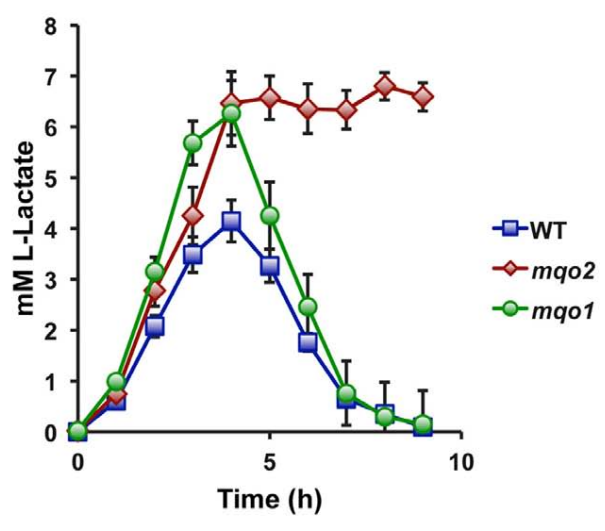

abolishes the ability to utilize L-lactate. Cells were grown aerobically in chemically defined medium with $0.2 \%$ L-lactate as the primary carbon source. Residual growth on medium components was subtracted out of depicted curves (see Materials and Methods). (C) The L-lactate excreted following NO--exposure cannot be reassimilated in a $\Delta m q 02$ mutant. Cells were grown in chemically defined medium as described in Figure 1B.

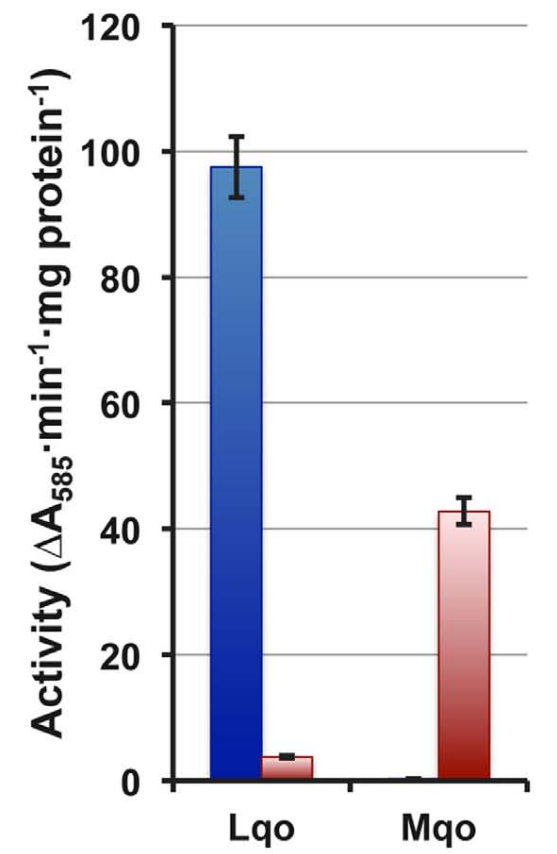

FIGURE 3 |The $S$. aureus Lqo enzyme oxidizes L-lactate and has no affinity for malate. Left: Membrane preparations from single mutant S. aureus were assayed for Lqo and Mqo activity. $\Delta m q 01$

(Lqo-containing) membranes react readily with L-lactate but not malate.

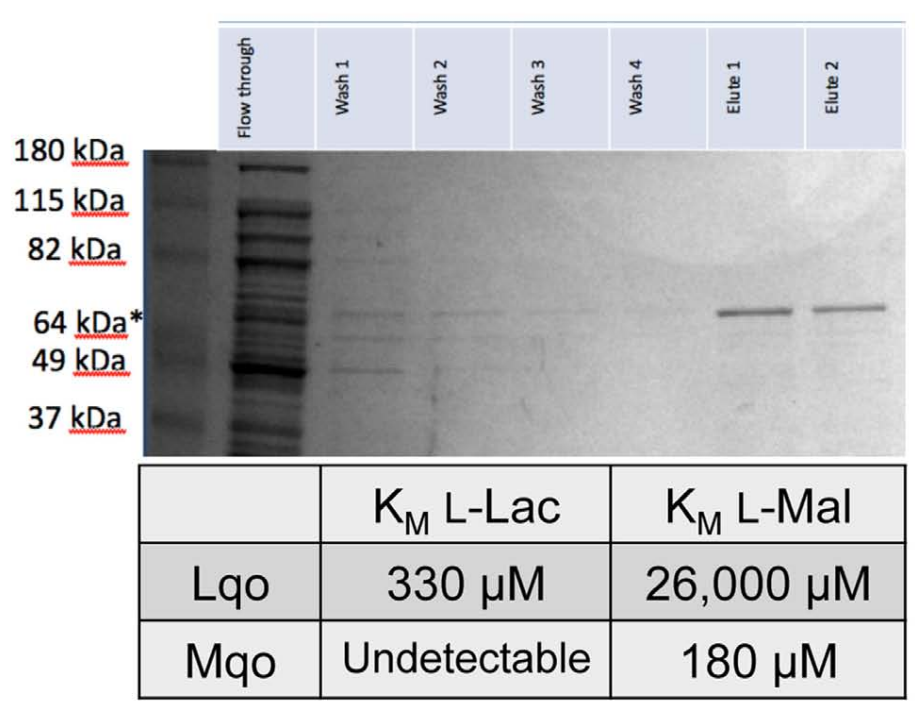

In contrast, $\Delta m q 02$ mutants (Mqo-containing) have activity against malate but not L-lactate. Right: Purified His6-tagged versions of Lqo and Mqo were used to determine affinities of each enzyme to its cognate substrate. 
L-lactate, we suggest that it be renamed Lqo (L-lactate-quinone oxidoreductase).

We assessed the physiological roles for Lqo in growth on various carbon sources in the presence/absence of different electron acceptors. As expected, mutants lacking Lqo showed measurable defects when grown on L-lactate and these defects were additive when $\Delta l q o$ was combined with $\Delta l d h 2$ (Figure A1 in Appendix). The loss of Ldh1 had little effect on L-lactate utilization consistent with its role in producing L-lactate for redox balance (Figure A1 in Appendix). When $S$. aureus was grown aerobically in media with amino acid carbon sources, Lqo was dispensable (Figure A2 in Appendix). In contrast a $\Delta m q o$ mutant was measurably impaired in this medium underscoring its role in the TCA cycle, a pathway essential to the utilization of amino acids (Figure A2 in Appendix). Regardless of the medium used to cultivate S. aureus, lqo transcript was highly abundant, though expression was significantly lower in the presence of glucose (Figure A3 in Appendix). Interestingly, lqo was still highly expressed in the $\Delta l d h 1 \Delta l d h 2$ mutant grown on glucose, an environment completely devoid of L-lactate (data not shown). Anaerobically, S. aureus was unable to grow on L-lactate as a carbon source unless an electron acceptor (e.g., nitrate) was also present (Figure A4A in Appendix). This result highlights the respiratory nature of Lqo in that it reduces the quinone pool therefore necessitating the presence of a terminal electron acceptor such as oxygen or nitrate. Interestingly, $\Delta$ lqo $S$. aureus could still grow anaerobically on L-lactate in the presence of nitrate implying that Ldh1 and/or Ldh2 can also serve as catabolic enzymes provided the resulting $\mathrm{NADH}$ is recycled through a respiratory mechanism (Figure A4A in Appendix). Moreover, combining peptides (1\% Tryptone) with L-lactate greatly improved anaerobic growth both in the presence and absence of nitrate (Figure A4B in Appendix). However, in the absence of nitrate, the added benefit of peptides combined with L-lactate was independent of Lqo and required Ldh1/2 in line with the absence of a defined electron acceptor (Figure A4B in Appendix). In contrast, as with L-lactate alone (Figure A4A in Appendix), addition of nitrate facilitated Lqodependent growth on peptide/L-lactate in that the $\Delta l d h 1 \Delta l d h 2$ mutant grew nearly as well as WT (Figure A4B in Appendix). Collectively, these data show that Lqo plays a pivotal role in $S$. aureus metabolism when utilizing L-lactate specifically under respiratory conditions.

\section{STAPHYLOCOCCUS AUREUS LqO IS REQUIRED FOR FULL VIRULENCE IN A MURINE SEPSIS MODEL}

Infecting mice i.v. with $5 \times 10^{6} \mathrm{cfu}$ of $S$. aureus strain Newman results in reproducible weight loss over the first week of infection (Figure 4). Roughly half of the mice lost $\geq 30 \%$ original body weight within the first week post-inoculation and were sacrificed as per approved IACUC protocols. In contrast, mice infected with an isogenic $\Delta$ lqo mutant exhibited significantly $(p<0.01)$ slower weight loss kinetics with all of the mice maintaining $\geq 70 \%$ of their original body weight (Figure 4). In competitive i.v. infections with WT strain Newman, the $\Delta$ lqo mutant showed a marked defect in the heart, but only a modest $\sim 3$-fold attenuation in the kidney (Figure 4). In mice unable to produce inflammatory NO. $\left(\mathrm{iNOS}^{-/-}\right)$, the heart-specific competitive defect was

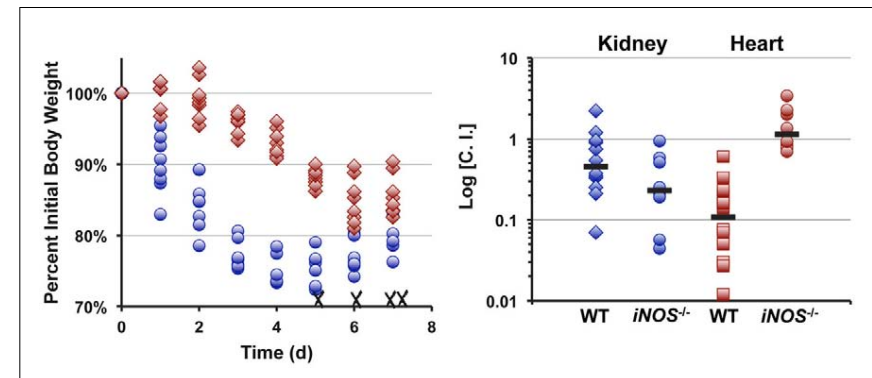

FIGURE 4 | Lqo is required for full $S$. aureus virulence in a murine sepsis model, particularly in establishing myocarditis. Left: Eight 4-to 6-week female C57BL/6 mice (two independent experiments each with four mice per group) were inoculated i.v. with $5 \times 10^{6} \mathrm{cfu}$ of $S$. aureus strain Newman (blue circles) or isogenic $\Delta /$ qo (red diamonds). Weight loss was monitored following inoculation and presented as percent initial body weight. Once mice lose $\geq 30 \%$ of their original body weight, they are sacrificed as per IACUC protocol. WT infected mice exhibited significantly greater weight loss ( $p<0.01$, using longitudinal linear regression to model weight loss over time, SAS ${ }^{\circledR}$, Cary, NC, USA) and four (50\%) were sacrificed (indicated with X). Right: Competitive indices (C.I.) were established by infecting mice i.v. with $1 \times 10^{7}$ cfu of a 1:1 mixture of WT: $\Delta$ /qo $S$. aureus strain Newman. Organs were harvested 5 days post-inoculation and mutant:WT C.I. ratios were calculated as outlined in experimental procedures. In parallel, isogenic $\mathrm{NOS}^{-/}$mice unable to produce inflammatory $\mathrm{NO}$. were also infected in a similar manner.

fully reversed while the modest defect in the kidneys was still observable (Figure 4). Thus, S. aureus Lqo contributes to virulence in both an $i N O S$-dependent and -independent fashion and mutants exhibit tissue-specific defects in competition with WT S. aureus.

The fact that the $\Delta l q o$ virulence defect was partially reversed in $\mathrm{iNOS}^{-/-}$mice was surprising given the lack of an in vitro $\mathrm{NO}$-phenotype when the mutant was grown on glucose (data not shown). Glucose is generally used in defined medium for in vitro experiments because glycolytic carbon sources support $S$. aureus NO--resistance while gluconeogenic sources do not (i.e., pyruvate, lactate, peptides, Figure 5A). However, combining L-lactate with peptides will support $S$. aureus growth under NO--stress, and this phenomenon is specific for L-lactate versus D-lactate and dependent on Lqo (Figure 5A). Furthermore, providing the product of Lqo $(0.1 \%$ pyruvate) in combination with peptides also supported growth during $\mathrm{NO}$-stress and as expected this was independent of Lqo (data not shown). We therefore hypothesized that Lqo was necessary for growth under NO--stress in the murine heart because of its requirement for replication using the combination of peptides and L-lactate during NO--exposure, and not because of its role in reassimilation of excreted L-lactate during growth on glucose.

Bacteria enumerated from murine heart tissue are found within abscesses associated with the cardiac muscle (myocarditis) or in some cases with the pericardial space (pericarditis; Figure 5B). Hematogenous myocarditis/pericarditis occurs regularly in the murine sepsis model in that bacteria are consistently isolated from cardiac tissue, though visible abscesses are not always present. Histological examination of infected tissue revealed infiltration of many granulocytic cells as well as other distinct cell types 

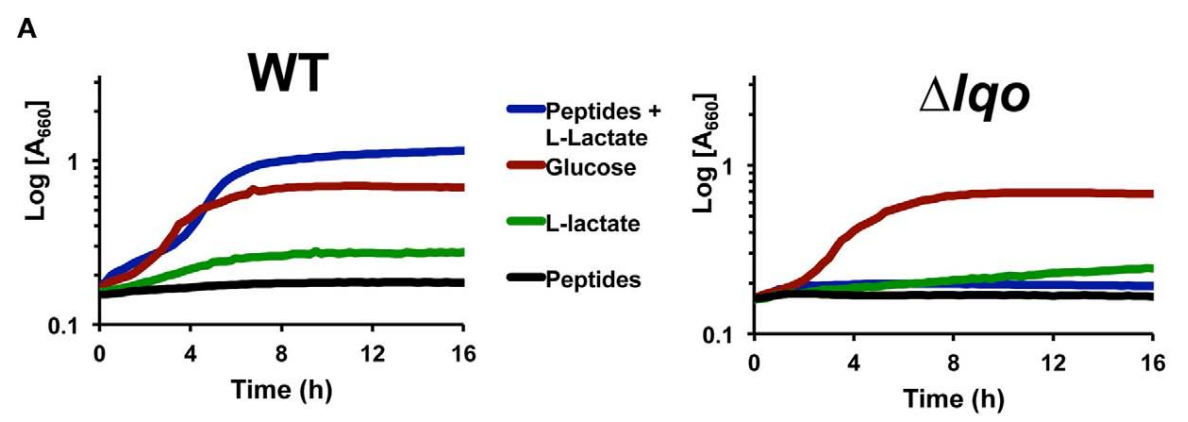

B

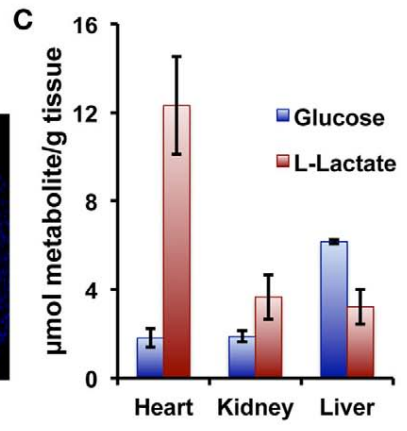

FIGURE 5 | Staphylococcus aureus growth in L-lactate-rich cardiac tissue in the presence of host NO. requires Lqo. (A) Individual gluconeogenic carbon sources (e.g., 1.0\% L-lactate or $1.0 \%$ tryptone) do not support NO. resistance in chemically defined medium as does $0.5 \%$ glucose. However, combining $0.1 \%$ L-lactate with $1.0 \%$ tryptone allows $S$. aureus to grow in the presence of NO. NO. in the form of $10 \mathrm{mM}$ DETA/NO was added once cells reach an $\mathrm{OD}_{660}=0.15$ depicted here as $t_{0}$. (B) Myocardial abscesses in mice infected with WT $S$. aureus Newman 5 days post-inoculation. Left, hematoxylin and eosin staining of abscess reveal a central bacterial microcolony (black arrow) surrounded by infiltrating granulocytic cells. Center, immuno-histofluorescent staining of the same abscess with anti-iNOS monoclonal Ab reveals robust iNOS expression throughout the abscess. Tissue was counter-stained with DAPI to reveal host cell nuclei. Non-staining S. aureus microcolony is visible (white arrow). Right, identical immuno-histofluorescent staining of a similar abscess in an iNOS $^{-/}$mouse as a negative control for anti-iNOS Ab. Tissue was also counter-stained with DAPI. (C) Metabolite data from murine tissues were determined using enzymatic methods previously described and normalized to total organ weight. surrounding micro-colonies of bacteria (Figure 5B). Immunohistological examination using anti-iNOS antibodies confirmed high-level iNOS expression in myocardial abscesses around $S$. aureus micro-colonies (Figure 5B). In comparison to other tissue commonly colonized hematogenously during $S$. aureus sepsis (e.g., hepatic and renal), cardiac tissue had by far the highest levels of L-lactate in addition to protein (Figure 5C). This is likely true for all active muscle tissue in mammals and is consistent with the specific requirement of Lqo for myocarditis/pericarditis in NO-proficient hosts.

\section{DISCUSSION}

The existence of NAD-independent lactate dehydrogenase ( $i \mathrm{LDH})$ activity in S. aureus was first described over 50 years ago, but received little attention over the NAD-dependent enzymes (Stockland and San Clemente, 1969). The S. aureus $i \mathrm{LDH}$ activity was again reported 4 years later and shown to be specific for L-lactate (Stevenson and Holdsworth, 1973). Subsequently, this $i$ LDH activity was shown to be membrane associated and was linked to the reduction of ferric iron, nitrate, or oxygen as terminal electron acceptors (Theodore and Weinbach, 1974; Lascelles and Burke, 1978; Tynecka and Malm, 1995). However, pure fractions containing only the $S$. aureus $i \mathrm{LDH}$ were never used to identify the protein. Thus, prior to this work, the gene(s) encoding the S. aureus $i \mathrm{LDH}$ was never identified, nor was this activity shown to be biologically relevant. We that the $\Delta l d h 1 \Delta l d h 2$ mutant, which does not produce any L-lactate, was still able to grow on L-lactate as a sole carbon source (Figure 1). Since lactate-racemic activity has been reported in a singular strain of $S$. aureus (Stockland and San Clemente, 1969), the triple $\Delta l d h 1 \Delta l d h 2 \Delta d d h$ mutant was tested and shown to still utilize L-lactate. Thus, the $\Delta l d h 1 \Delta l d h 2$ mutant was not racemically converting L-lactate to D-lactate and catabolizing the latter via Ddh. These data are consistent with $S$. aureus encoding a true $i \mathrm{LDH}$ that will promote growth on L-lactate as a carbon/energy source.

The ability of the $\Delta l d h 1 \Delta l d h 2$ mutant to utilize L-lactate was used to screen two annotated malate-quinone oxidoreductases for their role in promoting growth on L-lactate given its chemical similarity with malate. Since malate and lactate utilizing enzymes are closely related and relatively few amino acid changes can distinguish the two activities (Yin and Kirsch, 2007), it seemed reasonable that either mqo1 or mqo 2 encoded the $S$. aureus $i \mathrm{LDH}$. Mqo2 alone was shown to promote growth of the $\Delta l d h 1 \Delta l d h 2$ mutant on L-lactate thereby comprising the $S$. aureus $i \mathrm{LDH}$ activity (Figure 2). The enzyme catalyzes the oxidation of L-lactate (but not malate) with the concomitant reduction of menaquinone, thus we suggest renaming the enzyme Lqo (Figure 3). On the other hand, Mqo1 was shown to exhibit activity toward malate 
alone and is the only enzyme in S. aureus capable of oxidizing malate to oxaloacetate, the penultimate reaction of the TCA cycle (Figure 3). Accordingly, Mqo is essential for maximal growth on amino acid carbon sources since their assimilation through the TCA cycle to oxaloacetate is necessary for gluconeogenesis (Figure A2 in Appendix). Furthermore, $\Delta m q o 1$ mutants excrete excess lactate and acetate consistent with the lack of a functional TCA cycle resulting in "spill-over" metabolism at the pyruvate node (Figure $2 \mathrm{C}$ ). In contrast, $\Delta$ lqo mutants do not excrete excess acetate since the TCA cycle is fully intact (data not shown). Thus, Mqo and Lqo are enzymes with distinct, non-overlapping substrates and functions. Notably, both enzymes are essential for full virulence and $\Delta m q o$ mutants are severely attenuated underscoring the importance of the TCA cycle for $S$. aureus pathogenesis (Somerville et al., 2002; Figure A5 in Appendix).

At first glance, it seems illogical to express both Ldh1 and Lqo under NO--stress because they would represent futile cycles. However, given that the electron carriers differ between the Ldh enzymes and $i \mathrm{LDH}$, their net reaction resembles that of an alternative (non-proton pumping) NADH-dehydrogenase:

$$
\begin{array}{llll}
\text { Ldh1/Ldh2 } & \text { pyruvate }+\mathrm{NADH} & \leftrightarrow & \text { L-lactate }+\mathrm{NAD}^{+} \\
\text {Lqo } & \text { L-lactate }+\mathrm{MQ}=\mathrm{O} & \rightarrow & \text { pyruvate }+\mathrm{MQ}-\mathrm{OH} \\
& \mathrm{NADH}+\mathrm{MQ}=\mathrm{O} & \rightarrow & \mathrm{NAD}^{+}+\mathrm{MQ}-\mathrm{OH}
\end{array}
$$

Perhaps their dual expression provides $S$. aureus with an "uncoupled complex I" that can regenerate $\mathrm{NAD}^{+}$without affecting the proton-motive force. Interestingly, Lqo is still highly expressed in the $\Delta l d h 1 \Delta l d h 2$ mutant grown on glucose, a scenario where there is no L-lactate in the environment at all (none provided exogenously and none produced endogenously from glucose). The constitutive expression of Lqo regardless of the presence/absence of L-lactate implies that in the host environment, $S$. aureus always requires Lqo activity eliminating the need for a complex regulatory system to control transcription.

When S. aureus is grown on L-lactate as a carbon source, the role for Lqo is clearly evident. Mutants lacking Lqo exhibit slower growth and lower overall yields on L-lactate (Figure A1 in Appendix). This phenomenon is exacerbated in the $\Delta l d h 2$ background and not affected by deletion of $l d h 1$ implying that the major enzymes for L-lactate catabolism in S. aureus are Lqo and Ldh2 (Figure A1 in Appendix). Anaerobically, S. aureus cannot ferment L-lactate as a sole carbon source consistent with the requirement of a terminal electron acceptor for Lqo function and the fact that the reversal of Eq. 1 by Ldh 1 or Ldh 2 without a functioning respiratory chain would lead to redox imbalance (i.e., high NADH, Figures 6 and Figure A4A in Appendix). Simply providing nitrate as an electron acceptor allows $S$. aureus to grow anaerobically on L-lactate using either Lqo or Ldh1/Ldh2 (Figures 6 and Figure A4A in Appendix). The NAD-dependent LDHs (Ldh1 and Ldh2) catabolize L-lactate by the reversal of Eq. 1 presumably coupled to a respiratory $\mathrm{NADH}$-dehydrogenase. Interestingly, adding peptides to L-lactate-containing media allowed for growth anaerobically in the absence of nitrate and this was dependent on Ldh1/Ldh2 (Figure A4B in Appendix). Presumably, excess carbon from peptides frees up L-lactate-derived carbon to establish redox balance. For instance, some pyruvate generated from L-lactate-oxidation could be used for energy production while the rest could be converted to ethanol providing redox balance. Whatever the mechanism behind peptide stimulated growth under anaerobiosis, Lqo is not involved because it directly requires active respiration to generate oxidized quinone for enzymatic activity (i.e., the $\Delta$ lqo mutant grows as well as wild type without nitrate, Figure A4B in Appendix). Thus, S. aureus has evolved multiple means of assimilating L-lactate under various conditions implying its central role in this pathogen's metabolic environment. Moreover, Lqo appears to provide $S$. aureus with an efficient means of L-lactate catabolism in the presence of aerobic/anaerobic respiration.

The ability of $S$. aureus to resist host NO is key to its pathogenesis as demonstrated by the iNOS-dependent attenuation of the $\Delta$ lqo mutant, particularly in L-lactate-rich cardiac tissue (Figure 5). How S. aureus continues to catabolize host tissue in the presence of NO given the numerous metabolic targets of this immune radical is still unclear. However, here we demonstrate that Lqo provides another NO--resistant pathway to $S$. aureus when growing in a peptide/L-lactate-rich environment. Unlike glucose, individual gluconeogenic substrates do not allow $S$. aureus to circumvent the metabolic constraints imposed by exogenous NO. (Figure 5A). The reasons behind this phenomenon are unclear, but perhaps the multiple TCA cycle targets of NO limit growth on amino acids. However, combining L-lactate with peptides does restore $\mathrm{NO} \cdot$-resistance to $S$. aureus in an Lqo-dependent fashion, though the physiological reasons are still unclear (Figure 5A). D-lactate was unable to support growth under NO--stress in combination with peptides whereas pyruvate could independently of Lqo (data not shown). These data are consistent with the requirement for pyruvate combined with peptides to allow NO-resistant growth in the absence of glucose. It should be noted that the difference in NO-resistance between WT and $\Delta$ lqo in peptide/L-lactate medium was more robust with a slow releasing NO--donor such as DETA/NO. There were reproducible differences between WT and $\Delta$ lqo growth in peptide/L-lactate medium under high NO. levels $(\sim 1 \mathrm{mM}$ as in Figure 1), but they manifested as extended lag phases rather than altered growth rates (data not shown). This is consistent with the requirement of Lqo for some level of respiratory activity involving a terminal electron acceptor. At millimolar NO--levels, nearly all measurable respiration, both aerobic and anaerobic, is inhibited. However as NO. dissipates to sub-millimolar levels (Figure 1B), S. aureus may be able to respire using molecular oxygen mediated by a NO--resistant aerobic respiratory system. Indeed, Lqo-dependent utilization of L-lactate/peptides in the presence of $\mathrm{NO}$. was eliminated in the $\triangle$ qoxBACD mutant implying that the cytochrome- $a a_{3}$ quinol oxidase is essential for Lqo activity (Figure A6 in Appendix). While the genes encoding the cytochrome $b d$ quinol oxidase are induced by NO. (Richardson et al., 2006; Hochgrafe et al., 2008), this oxidase does not seem to support growth on Llactate/peptides during NO--stress (Figure A6 in Appendix). The requirement for Lqo during infection of murine cardiac tissue implies that $\mathrm{NO} / \mathrm{O}_{2}$ levels are likely amenable to active cytochrome- $a a_{3}$ supporting Lqo-dependent L-lactate utilization. Alternatively, during infection $S$. aureus may utilize flavohemoglobin (Hmp)-derived nitrate from $\mathrm{NO}$-detoxification as a 
terminal electron acceptor supporting Lqo-mediated L-lactate utilization. More work is required to elucidate the nature of the relevant $S$. aureus electron acceptor under NO--stress during infection.

The fact that either Lqo or Ldh1/2 can support growth on $\mathrm{L}^{-}$ lactate anaerobically with nitrate represents a significant difference from the exclusive requirement for Lqo during L-lactate/peptide utilization under NO. stress (Figure 6). For Ldh1/2 to catabolize L-lactate via the reversal of Eq. 1, a respiratory system must be present to recycle NADH. Since respiration is also required for Lqo function during NO--stress, that same respiratory activity should be able to recycle the NADH produced by Ldh1/2. This is not the case however, thus NO. must inhibit some other aspect of oxidative phosphorylation unique to the direct recycling of $\mathrm{NADH}$. One explanation is that NO may inhibit the NADHdehydrogenase expressed during NO--exposure (Figure 6). This would not affect Lqo activity but would limit the utility of Ldh1/2 even in the presence of a usable electron acceptor. Future experimentation will be directed at defining the effects of NO. that necessitate Lqo activity specifically for the utilization of L-lactate during NO--stress.

While the role of Lqo in NO--resistant L-lactate/peptide catabolism raises important metabolic questions, its disproportionate contribution to virulence in tissues with excess L-lactate (e.g., cardiac muscle tissue) has intriguing implications regarding S. aureus pathogenesis. While muscle tissue can have excessive amounts of

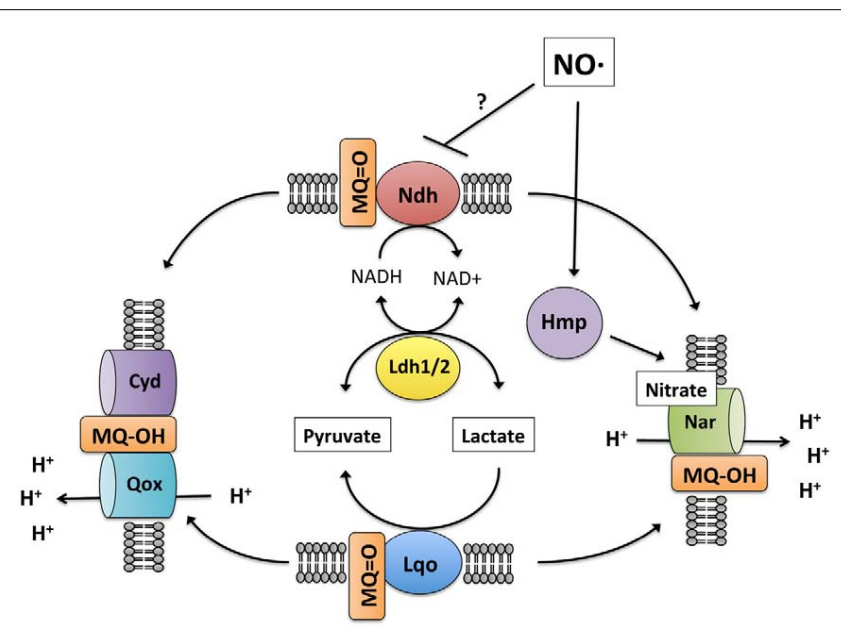

FIGURE 6 | Model of $\boldsymbol{S}$. aureus lactate metabolism. During respiratory lactate catabolism (either aerobically or anaerobically on nitrate), S. aureus can use either Lqo or reversal of the NADH-dependent Ldh1/2 provided $\mathrm{NADH}$ can be recycled. In the absence of an external electron acceptor, $S$. aureus is unable to catabolize lactate because Lqo activity requires a functioning quinol oxidase as does $L d h 1 / 2$ for $N A D^{+}$regeneration. Initially during NO. stress, respiration is inhibited and lactate is produced for redox balance (NAD+ generation). Once respiration resumes due to waning NO. levels, lactate can be assimilated but only in an Lqo-dependent fashion. Despite the presence of active quinol oxidases (Cox, Cyd, or Nar) $\mathrm{NADH}$-dependent Ldh1/2 do not promote lactate assimilation in the presence of NO. This would be consistent with NO--dependent inhibition of $\mathrm{Ndh}$, limiting $\mathrm{NAD}^{+}$regeneration even in the presence of active quinol oxidases, though this remains to be demonstrated.
L-lactate given the oxygen demand associated with muscle activity, other mammalian tissues relevant to $S$. aureus biology are also replete with high levels of L-lactate. For instance, human sweat can have as high as $\sim 50 \mathrm{mM}$ L-lactate, possibly resulting in a role for Lqo in skin colonization (Sakharov et al., 2010). Moreover, nasal secretions have also been shown to contain high concentrations of L-lactate making the most common site of $S$. aureus colonization (the nasal cavity) a prime environment for Lqo activity (Stierna et al., 1991; Westrin et al., 1992). Thus, while Lqo may contribute to $S$. aureus disease, particularly in various forms of myocarditis and myositis, this newly characterized enzyme may also have more overarching roles in promoting S. aureus colonization. Thus, the identification of Lqo as comprising the elusive $S$. aureus $i \mathrm{LDH}$ activity will provide significant insight into the physiology, pathogenesis, and general biology of this very significant human pathogen.

\section{MATERIALS AND METHODS BACTERIAL STRAINS AND GROWTH CONDITIONS}

Staphylococcus aureus was cultivated in Brain Heart Infusion medium or in chemically defined medium (PN medium) in which primary carbon sources could be modified (Pattee, 1976). Briefly, PN medium is a phosphate-buffered medium composed of a primary carbon source (variable depending on experiment), nucleobases (Adenine, $5 \mathrm{mg} / \mathrm{L}$; Guanine, $5 \mathrm{mg} / \mathrm{L}$; Cytosine, $5 \mathrm{mg} / \mathrm{L}$; Uracil, $5 \mathrm{mg} / \mathrm{L}$; and Thymine, $20 \mathrm{mg} / \mathrm{L}$ ), free amino acids (Ala, 60 mg/L; Arg, 70 mg/L; Asp, 90 mg/L; Cystine, 20 mg/L; Glu $100 \mathrm{mg} / \mathrm{L}$; Gly, $50 \mathrm{mg} / \mathrm{L}$; His, $30 \mathrm{mg} / \mathrm{L}$; Iso, $30 \mathrm{mg} / \mathrm{L}$; Leu, 90 mg/L; Lys, 70 mg/L; Met, 10 mg/L; Phe, 40 mg/L; Pro, 10 mg/L; Ser, $30 \mathrm{mg} / \mathrm{L}$; Thr, $30 \mathrm{mg} / \mathrm{L}$; Trp, $10 \mathrm{mg} / \mathrm{L}$; Tyr, $50 \mathrm{mg} / \mathrm{L}$; and Val, $80 \mathrm{mg} / \mathrm{L}$ ), vitamins (thiamine, $1 \mathrm{mg} / \mathrm{L}$; niacin, $1.2 \mathrm{mg} / \mathrm{L}$; biotin, $5 \mu \mathrm{g} / \mathrm{L}$; and pantothenate, $250 \mu \mathrm{g} / \mathrm{L}), \mathrm{FeCl}_{3}$ at $8 \mathrm{mg} / \mathrm{L}, \mathrm{MgSO}_{4}$ at $2.5 \mathrm{mg} / \mathrm{L}$, and trace elements $(\mathrm{ZnCl}, 70 \mu \mathrm{g} / \mathrm{L} ; \mathrm{MnCl}, 63 \mu \mathrm{g} / \mathrm{L}$; Boric Acid, $6 \mu \mathrm{g} / \mathrm{L} ; \mathrm{CoCl}_{2}, 190 \mu \mathrm{g} / \mathrm{L} ; \mathrm{CuCl}_{2}, 2 \mu \mathrm{g} / \mathrm{L} ; \mathrm{NiCl}_{2}, 13 \mu \mathrm{g} / \mathrm{L}$; and $\left.\mathrm{Na}_{2} \mathrm{MoO}_{4}, 31 \mu \mathrm{g} / \mathrm{L}\right)$. Aerobic growth was achieved by shaking $(250 \mathrm{rmp})$ in flasks or slanted test tubes where as anaerobic cultivation was performed in a Coy anaerobe chamber. Antibiotic selection in $S$. aureus (E. coli) was performed using the following concentrations: ampicillin $(100 \mu \mathrm{g} / \mathrm{ml})$, chloramphenicol $20 \mu \mathrm{g} / \mathrm{ml}$, kanamycin $50 \mu \mathrm{g} / \mathrm{ml}(50 \mu \mathrm{g} / \mathrm{ml})$, spectinomycin $100 \mu \mathrm{g} / \mathrm{ml}(500 \mu \mathrm{g} / \mathrm{ml})$, erythromycin $5 \mu \mathrm{g} / \mathrm{ml}(300 \mu \mathrm{g} / \mathrm{ml})$ unless otherwise indicated. Growth was monitored as change in absorbance $(660 \mathrm{~nm})$ assessed using a Tecan infinite M200 plate reader in $200 \mu \mathrm{l}$ cultures within a 96 well plate. To assess the inability to grow in defined media with $\mathrm{L}$ - or D-lactate as a primary carbon source, residual growth on medium components was subtracted from growth in media replete with lactate carbon sources. Residual growth in media lacking primary carbon sources halted at $\mathrm{OD}_{660}=0.2-0.3$. Mutations were constructed using a modified allelic exchange method involving the cloning of flanking DNA sequence on either side of a selectable marker in the $S$. aureus/E. coli shuttle vector, pBT2ts. This construct was electroporated into $S$. aureus as previously described and grown with selection at $30^{\circ} \mathrm{C}$. Cointegration was achieved by growing cultures overnight with selection at $43^{\circ} \mathrm{C}$ followed by plating on antibiotic containing media (Bruckner, 1997). Single colonies were isolated from $43^{\circ} \mathrm{C}$ and grown overnight for three consecutive days 
without antibiotic selection at $30^{\circ} \mathrm{C}$. Finally, cultures were diluted 1:100 and grown to mid-exponential phase at $37^{\circ} \mathrm{C}$ at such time chloramphenicol was added $(10 \mu \mathrm{g} / \mathrm{ml})$ to inhibit the growth of resolved cointegrates. After $30 \mathrm{~min}$, cycloserine was administered $(100 \mu \mathrm{g} / \mathrm{ml})$ to selectively kill $\mathrm{Cm}^{\mathrm{R}}$-cointegrates. After $\geq 4 \mathrm{~h}$ incubation at $37^{\circ} \mathrm{C}$, surviving cells are plated on selective media and screened for successful allelic replacement.

\section{VIRULENCE ASSESSMENT}

Four to 6-week-old female C57BL/6 mice were inoculated i.v. via tail vein with $5 \times 10^{6} \mathrm{cfu}$ in $100 \mu \mathrm{l}$ of $S$. aureus strain Newman or isogenic mutants. Weight loss was monitored daily for 1 week as a metric for disease progression, mice exhibiting $\geq 30 \%$ weight loss were sacrificed as per IACUC approved protocol. Alternatively, WT mice or isogenic iNOS ${ }^{-1-}$ mice (Jackson Laboratories) were similarly infected with $1 \times 10^{7} \mathrm{cfu}$ of WT $S$. aureus strain Newman at a 1:1 mixture with isogenic $\Delta$ lqo and tissue harvested 5 days post-inoculation. Kidneys and hearts were extracted, and ratios of $\Delta$ lqo:WT were determined by plating on selective and non-selective media. Competitive indices (C.I.s) were calculated as $\mathrm{d} 5$ organ burden ratio $(\Delta l q o / \mathrm{WT})$ divided by inoculation ratio $(\Delta$ lqo/WT).

\section{ENZYMATIC DETERMINATION OF METABOLITE LEVELS}

Metabolites such as L- and D-lactate, glucose and nitrate were quantified as previously described as per manufacturer instruction (Richardson et al., 2008). Excreted metabolite levels from $S$. aureus cultures were determined using supernatants from $1 \mathrm{ml}$ of pelleted, heat-inactivated $\left(70^{\circ} \mathrm{C}\right.$ for $\left.5 \mathrm{~min}\right)$ cells. Tissue metabolites were extracted from organs (liver, heart, kidneys) harvested immediately from individual mice (in triplicate) following euthanization via cervical dislocation as per approved IACUC protocols. The organs were quickly homogenized in $\mathrm{PBS}$ at $4^{\circ} \mathrm{C}$, quenched with ice cold methanol (final $80 \% \mathrm{v} / \mathrm{v}$ ) and frozen at $-80^{\circ} \mathrm{C}$. Subsequently, tissues were thawed and metabolites were extracted by adding chloroform and water to achieve a final ratio methanol:chloroform:water (4:4:3). The aqueous phase was

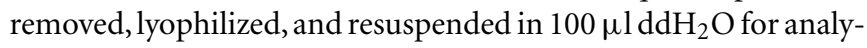
ses. Lactate and glucose levels were normalized to organ weight (mg tissue).

\section{FLUORESCENT IMMUNOHISTOCHEMISTRY}

Immunohistochemistry on heart tissues was performed as previously described with minor modifications (Thurlow et al., 2011). Heart tissue was collected 5 days post-inoculation and fixed in $10 \%$ formalin then paraffin embedded and sectioned $(10 \mu \mathrm{m})$ by the Histopathology Core Facility at UNC. Sections were deparaffinized using a graded series of xylene and ethanol washes followed by microwaving for $20 \mathrm{~min}$ in $10 \mathrm{mM} \mathrm{NaCl}$ buffer ( $\mathrm{pH} \mathrm{6}$ ) for antigen retrieval. Tissue sections were stained with primary rabbitderived antibodies against iNOS (Abcam, Cambridge, MA, USA) and secondary donkey anti-rabbit biotin conjugated antibodies (Jackson ImmuoResearch Laboratories, West Grove, PA, USA) followed by the addition of DyLight ${ }^{\mathrm{TM}} 594$-conjugated streptavidin (Jackson ImmunoResearch). Images were acquired using a Leica SP2 confocal microscope (Leica Microsystems, Buffalo Grove, IL, USA).

\section{MEMBRANE PREPARATIONS AND Lqo/Mqo ENZYMATIC ASSAYS}

Overnight $S$. aureus cultures ( $\Delta l q o$ to measure Mqo activity, $\Delta m q o$ to determine Lqo activity, and $\Delta m q o \Delta l q o$ for background activity) were diluted 1:100 in $100 \mathrm{ml}$ of $\mathrm{LB}$ medium (low glucose to prevent Mqo repression) and shaken at $37^{\circ} \mathrm{C}$ until $\mathrm{OD}_{660}=\sim 0.8$. Cells were pelleted, washed in PBS then incubated at $37^{\circ} \mathrm{C}$ in the presence of Lysostaphin $(12.5 \mu \mathrm{g} / \mathrm{ml})$, Lysozyme $(12.5 \mu \mathrm{g} / \mathrm{ml})$, DNase $(10 \mu \mathrm{g} / \mathrm{ml})$, and RNase $(10 \mu \mathrm{g} / \mathrm{ml})$ for $1 \mathrm{~h}$. Debris was pelleted and supernatants ultracentrifuged at 100,000 $\mathrm{g}$ for $1 \mathrm{~h}$ at $4^{\circ} \mathrm{C}$. Membrane fraction pellets were resuspended then sonicated and protein was quantified using BCA procedure (Pierce). Reaction mixtures contain $1 \mathrm{mg}$ total membrane protein, $40 \mu \mathrm{g}$ nitro blue tetrazolium (NBT), $5 \mathrm{mM} \mathrm{KCN}$, and were initiated by addition of $20 \mathrm{mM}$ substrate (malate/L-lactate) in a final volume of $200 \mu$ l. Reaction progress is monitored by measuring absorbance at $585 \mathrm{~nm}$ every $30 \mathrm{~s}$ for 20 min using a Tecan infinite M200 plate reader.

\section{Lqo AND Mqo PURIFICATION AND ANALYSES}

C-terminal His $_{6}$-tagged versions of Lqo and Mqo were constructed by amplifying alleles from $S$. aureus strain COL chromosomal DNA using primers Mqo2_His.1A/1B and Mqo1_His.1A/1B, respectively, and cloning into the NcoI/BamHI sites of pQE-60 (Qiagen). Resulting constructs (pJF132 and pJF133) were transformed into E. coli M15 (pREP4; Qiagen) maintained at $37^{\circ} \mathrm{C}$ with kanamycin $(25 \mu \mathrm{g} / \mathrm{ml})$ and ampicillin $(200 \mu \mathrm{g} / \mathrm{ml})$. For purification, $1 \mathrm{~L}$ cultures were seeded with $20 \mathrm{~mL}$ overnight cultures, and grown at $25^{\circ} \mathrm{C}$ for $4 \mathrm{~h}$ with selection and the addition of IPTG $(0.1 \mathrm{mM})$. Subsequently, cells were pelleted, washed in PBS and lysed via sonification. Enzymes were purified using HisPur Cobalt Purification System (Pierce) as per manufacturer instruction. Elutions were dialyzed twice for $2 \mathrm{~h}$ at $4^{\circ} \mathrm{C}$ followed by an overnight dialysis at $4^{\circ} \mathrm{C}$ against high-salt $(300 \mathrm{mM} \mathrm{NaCl}) \mathrm{PBS}$. Yields were quantified using BCA Protein Quantification Kits (Pierce).

Enzymatic activity for Mqo and Lqo was monitored by addition of $100 \mathrm{ng}$ purified His6-Tagged protein ( $1 \mu \mathrm{g}$ if using non-specific substrate) in a $200 \mu \mathrm{l}$ reaction mixture consisting of $1.4 \mathrm{mM}$ menaquinone, $5 \mu \mathrm{M}$ flavin adenine dinucleotide (FAD), $643 \mu \mathrm{g}$ phosphatidylethanolamine (PE), $40 \mu \mathrm{g}$ NBT and $20 \mathrm{mM}$ substrate (malate/L-lactate). Prior to addition of enzyme, reaction mix was sonicated to uniformly disperse menaquinone within the PE vesicles. Reactions were initiated by addition of substrate and followed via monitoring absorbance at $585 \mathrm{~nm}$ using a Tecan infinite M200 plate reader.

RNA EXTRACTION AND QUANTITATIVE REVERSE-TRANSCRIPTASE PCR Cells were grown in PN chemically defined medium supplemented with either $0.5 \%$ glucose, $1.0 \%$ L-lactate, or $1.0 \%$ tryptone as primary carbon sources. Once cells reached $\mathrm{OD}_{660}=0.5,25 \mathrm{ml}$ of culture was added to $25 \mathrm{ml}$ of ice cold ethanol:acetone (1:1) and incubated at $-80^{\circ} \mathrm{C}$ until further use. Frozen cell suspensions were thawed at room temperature, pelleted by centrifugation and resuspended in $500 \mu \mathrm{l}$ of TE for mechanical disruption using Lysing Matrix B (MP Biomedicals, Solon, OH, USA) in a standard cell disruptor. Hundred microliter of lysates was used for RNA isolation using an RNAEasy ${ }^{\circledR}$ Mini Kit (Qiagen, Valencia, CA, USA) as per manufacturer instructions. 
RNA was spectrophotometrically quantified and $50 \mathrm{ng}$ of total RNA was analyzed per reaction using the OneStep ${ }^{\mathrm{TM}} \mathrm{SYBR}^{\circledR}$ RTPCR kit (Quantace, Valencia, CA, USA) on an iCycler Real Time Machine (BioRad). Primers used for analysis are listed in Table A1 in Appendix and both mqo and lqo transcript levels were normalized to those of $r p o D$.

\section{REFERENCES}

Bruckner, R. (1997). Gene replacement in Staphylococcus carnosus and Staphylococcus xylosus. FEMS Microbiol. Lett. 151, 1-8.

DeGroote, M. A., and Fang, F. C. (1999). Nitric Oxide and Infection, Vol. 1, ed. F. C. Fang (Kluwer Academic/Plenum Publishers), 231-247.

Diekema, D. J., Pfaller, M. A., Schmitz, F. J., Smayevsky, J., Bell, J., Jones, R. N., and Beach, M. (2001). Survey of infections due to Staphylococcus species: frequency of occurrence and antimicrobial susceptibility of isolates collected in the United States, Canada, Latin America, Europe, and the Western Pacific region for the SENTRY Antimicrobial Surveillance Program, 1997-1999. Clin. Infect. Dis. 32(Suppl. 2), S114-S132.

Foster, T. J. (2005). Immune evasion by staphylococci. Nat. Rev. Microbiol. 3, 948-958.

Hochgrafe, F., Wolf, C., Fuchs, S., Liebeke, M., Lalk, M., Engelmann, S., and Hecker, M. (2008). Nitric oxide stress induces different responses but mediates comparable protein thiol protection in Bacillus subtilis and Staphylococcus aureus. J. Bacteriol. 190, 4997-5008.

Klacsmann, P. G., Bulkley, B. H., and Hutchins, G. M. (1977). The changed spectrum of purulent pericarditis: an 86 year autopsy experience in 200 patients. Am. J. Med. 63, 666-673.

Klevens, R. M., Morrison, M. A., Nadle, J., Petit, S., Gershman, K., Ray, S., Harrison, L. H., Lynfield, R., Dumyati, G., Townes, J. M., Craig, A. S., Zell, E. R., Fosheim, G. E., McDougal, L. K., Carey, R. B., and Fridkin, S. K. (2007). Invasive methicillin-resistant Staphylococcus aureus infections in the United States. JAMA 298, 1763-1771.

Lascelles, J., and Burke, K. A. (1978). Reduction of ferric iron by L-lactate and DL-glycerol-3-phosphate in membrane preparations from Staphylococcus aureus and interactions with the nitrate reductase system. J. Bacteriol. 134, 585-589.

Madern, D. (2002). Molecular evolution within the L-malate and L-lactate dehydrogenase super-family. J. Mol. Evol. 54, 825-840.

Mermel, L. A., Cartony, J. M., Covington, P., Maxey, G., and Morse, D. (2011). Methicillin-resistant Staphylococcus aureus colonization at different body sites: a prospective, quantitative analysis. J. Clin. Microbiol. 49, 1119-1121.

Mookadam, F., Moustafa, S. E., Sun, Y., Wilson, F. C., Mohammed, S. S., Park, S., Tleyjeh, I. M., Oh, J. K., and Tajik, J. (2009). Infectious pericarditis: an experience spanning a decade. Acta Cardiol. 64, 297-302.

Pannaraj, P. S., Hulten, K. G., Gonzalez, B. E., Mason, E. O. Jr., and Kaplan, S. L. (2006). Infective pyomyositis and myositis in children in the era of community-acquired, methicillin-resistant Staphylococcus aureus infection. Clin. Infect. Dis. 43, 953-960.

Pattee, P. A. (1976). Genetic linkage of chromosomal tetracycline resistance and pigmentation to a purine auxotrophic marker and the isoleucinevaline-leucine structural genes in Staphylococcus aureus. J. Bacteriol. 127, 1167-1172.

Richardson, A. R., Dunman, P. M., and Fang, F. C. (2006). The nitrosative stress response of Staphylococcus aureus is required for resistance to innate immunity. Mol. Microbiol.61, 927-939.

Richardson, A. R., Libby, S. J., and Fang, F. C. (2008). A nitric oxide-inducible lactate dehydrogenase enables Staphylococcus aureus to resist innate immunity. Science 319, 1672-1676.

Richardson, A. R., Payne, E. C., Younger, N., Karlinsey, J. E., Thomas, V. C, Becker, L. A., Navarre, W. W., Castor, M. E., Libby, S. J., and Fang, F. C. (2011). Multiple targets of nitric oxide in the tricarboxylic acid cycle of Salmonella enterica serovar

\section{ACKNOWLEDGMENTS}

The authors would like to thank A. S. Richardson for assistance with statistical analyses. This work was supported by funding from the NIH NIAID (AI093613 to Anthony R. Richardson) and The Pew Charitable Trusts (Pew Scholars Program in the Biomedical Sciences).

Typhimurium. Cell Host Microbe 10, 33-43.

Richardson, A. R., Soliven, K. C., Castor, M. E., Barnes, P. D., Libby, S. J., and Fang, F. C. (2009). The base excision repair system of Salmonella enterica serovar Typhimurium counteracts DNA damage by host nitric oxide. PLoS Pathog. 5, e1000451. doi:10.1371/journal.ppat.1000451

Sakharov, D. A., Shkurnikov, M. U., Vagin, M. Y., Yashina, E. I., Karyakin, A. A., and Tonevitsky, A. G. (2010). Relationship between lactate concentrations in active muscle sweat and whole blood. Bull. Exp. Biol. Med. 150, 83-85.

Somerville, G. A., Chaussee, M. S., Morgan, C. I., Fitzgerald, J. R., Dorward, D. W., Reitzer, L. J., and Musser, J. M. (2002). Staphylococcus aureus aconitase inactivation unexpectedly inhibits post-exponential-phase growth and enhances stationaryphase survival. Infect. Immun. 70, 6373-6382.

Stevenson, P. M., and Holdsworth, E. S. (1973). The distribution of NADindependent lactic dehydrogenases amongst micro-organisms. J. Gen. Microbiol. 78, 83-88.

Stierna, P., Soderlund, K., and Hultman, E. (1991). Chronic maxillary sinusitis. Energy metabolism in sinus mucosa and secretion. Acto Otolaryngol. 111, 135-143.

Stockland, A. E., and San Clemente, C. L. (1969). Multiple forms of lactate dehydrogenase in Staphylococcus aureus. J. Bacteriol. 100, 347-353.

Theodore, T. S., and Weinbach, E. C. (1974). Respiratory activities associated with mesosomal vesicles and protoplast membranes of Staphylococcus aureus. J. Bacteriol. 120, 562-564.

Thurlow, L. R., Hanke, M. L., Fritz, T., Angle, A., Aldrich, A., Williams, S. H., Engebretsen, I. L., Bayles, K. W., Horswill, A. R., and Kielian, T. (2011). Staphylococcus aureus biofilms prevent macrophage phagocytosis and attenuate inflammation in vivo. $J$. Immunol. 186, 6585-6596.
Tynecka, Z., and Malm, A. (1995). Intrinsic insensitivity to cadmium of the L-lactate oxidizing system in Staphylococcus aureus. FEMS Microbiol. Lett. 129, 11-15.

Wasi, F., and Shuter, J. (2003). Primary bacterial infection of the myocardium. Front. Biosci. 8, s228s231.

Westrin, K. M., Stierna, P., Soderlund, K., and Carlsoo, B. (1992). Lactic acid isomers and fatty acids in sinus secretion: a longitudinal study of bacterial and leukocyte metabolism in experimental sinusitis. Scand. J. Infect. Dis. 24, 765-772.

Yin, Y., and Kirsch, J. F. (2007). Identification of functional paralog shift mutations: conversion of Escherichia coli malate dehydrogenase to a lactate dehydrogenase. Proc. Natl. Acad. Sci. U.S.A. 104, 17353-17357.

Conflict of Interest Statement: The authors declare that the research was conducted in the absence of any commercial or financial relationships that could be construed as a potential conflict of interest.

Received: 15 October 2011; paper pending published: 15 November 2011; accepted: 05 December 2011; published online: 27 December 2011.

Citation: Fuller JR, Vitko NP, Perkowski EF, Scott E, Khatri D, Spontak JS, Thurlow LR and Richardson AR (2011) Identification of a lactatequinone oxidoreductase in Staphylococcus aureus that is essential for virulence. Front. Cell. Inf. Microbio. 1:19. doi: 10.3389/fcimb.2011.00019

Copyright (C) 2011 Fuller, Vitko, Perkowski, Scott, Khatri, Spontak, Thurlow and Richardson. This is an open-access article distributed under the terms of the Creative Commons Attribution Non Commercial License, which permits non-commercial use, distribution, and reproduction in other forums, provided the original authors and source are credited. 


\section{APPENDIX}

Table A1 | Strains used in this work.

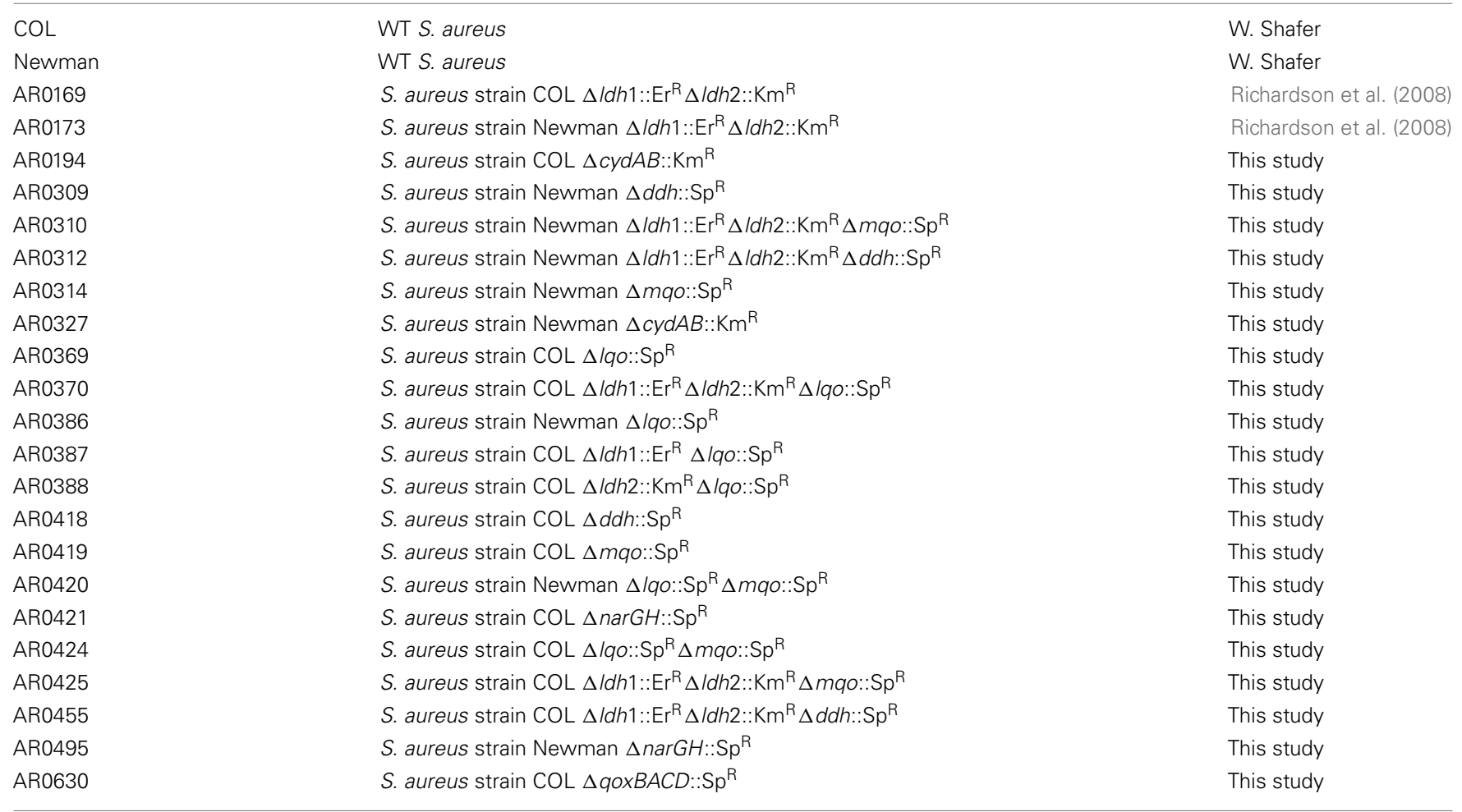

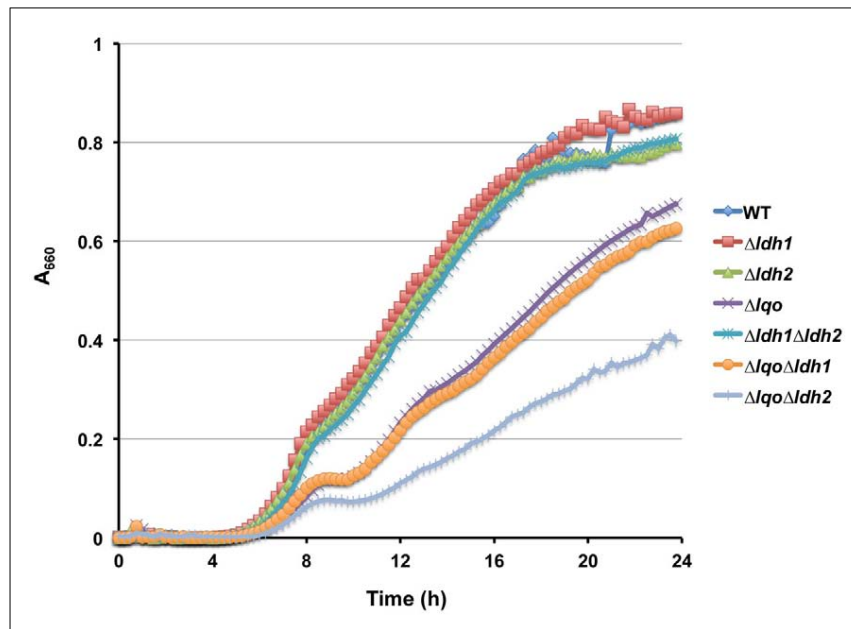

FIGURE A1 | Lqo, and to a lesser extent Ldh2, are required for maximal growth on L-lactate. Representative growth curve of WT $S$. aureus strain $\mathrm{COL}$ and isogenic mutants grown in chemically defined medium supplemented with $1.0 \%$ L-lactate as a primary carbon source. Growth as determined by absorbance at $660 \mathrm{~nm}$ was normalized to minimal growth in the absence of added carbon. 
Table A2 | Primers/plasmids used in this study.

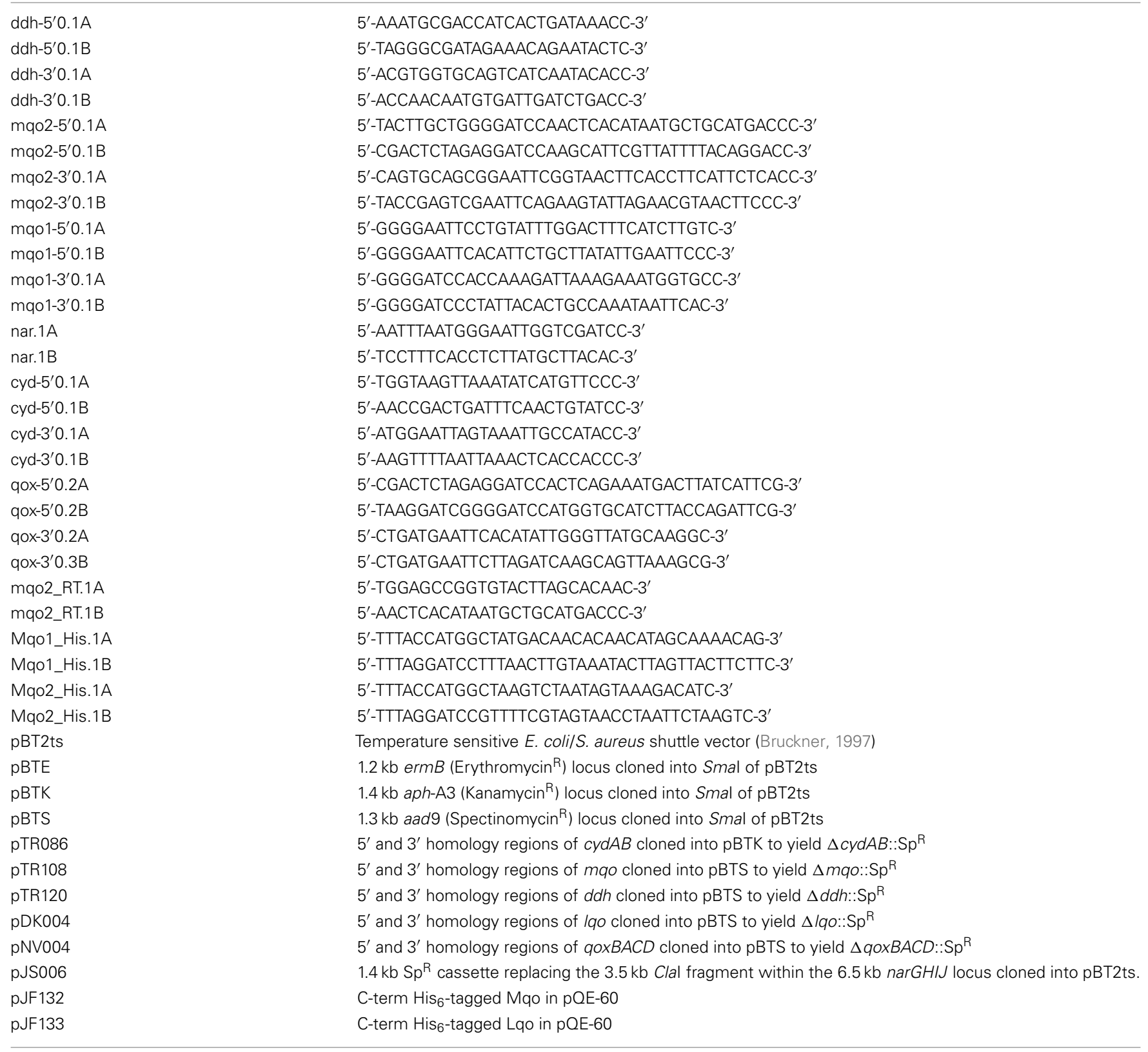



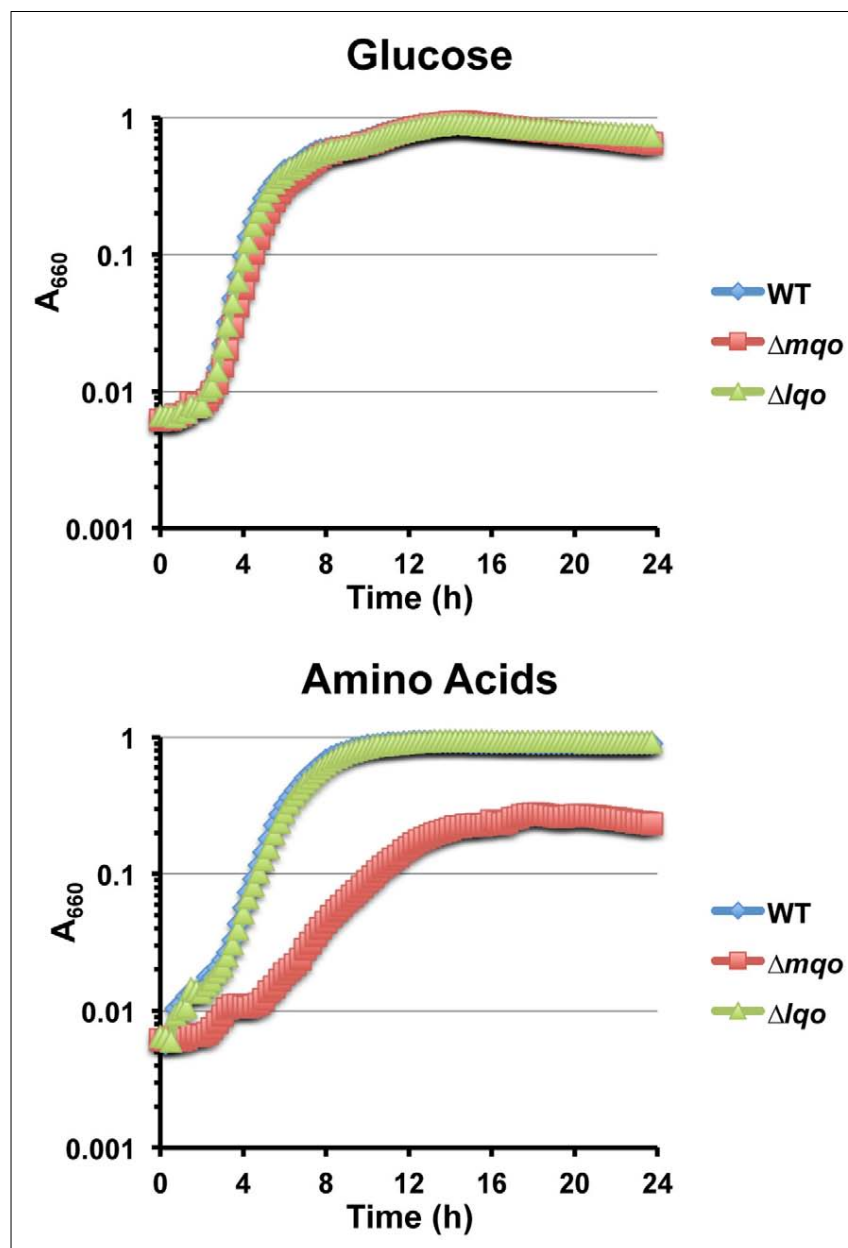

FIGURE A2 | Lqo and Mqo are differentially required for utilization of various carbon sources. TOP: Neither Mqo nor Lqo are essential for growth in chemically defined media using glucose $(0.5 \%)$ as a primary carbon source. BOTTOM: While Mqo is dispensable for growth on L-lactate (Figure 2) it is essential for maximal growth in chemically defined media using amino acids (0.5\% Cas Amino Acids) as a primary carbon source.

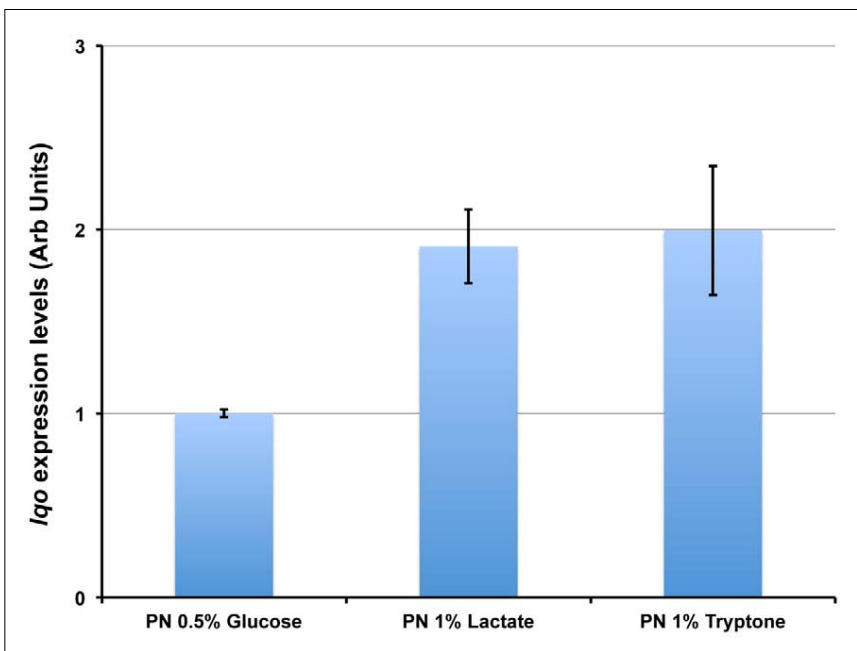

FIGURE A3 | lqo is highly expressed under a variety of culture conditions. Total RNA was extracted from $S$. aureus strain $\mathrm{COL}$ grown in chemically defined medium with either $0.5 \%$ Glucose, $1.0 \%$ L-lactate, or $1.0 \%$ tryptone as primary carbon sources. Quantitative RT-PCR was performed using primers specific for lqo and transcript levels were normalized to $r p o D$. Data are depicted relative to lqo transcript levels from cells grown on Glucose. 

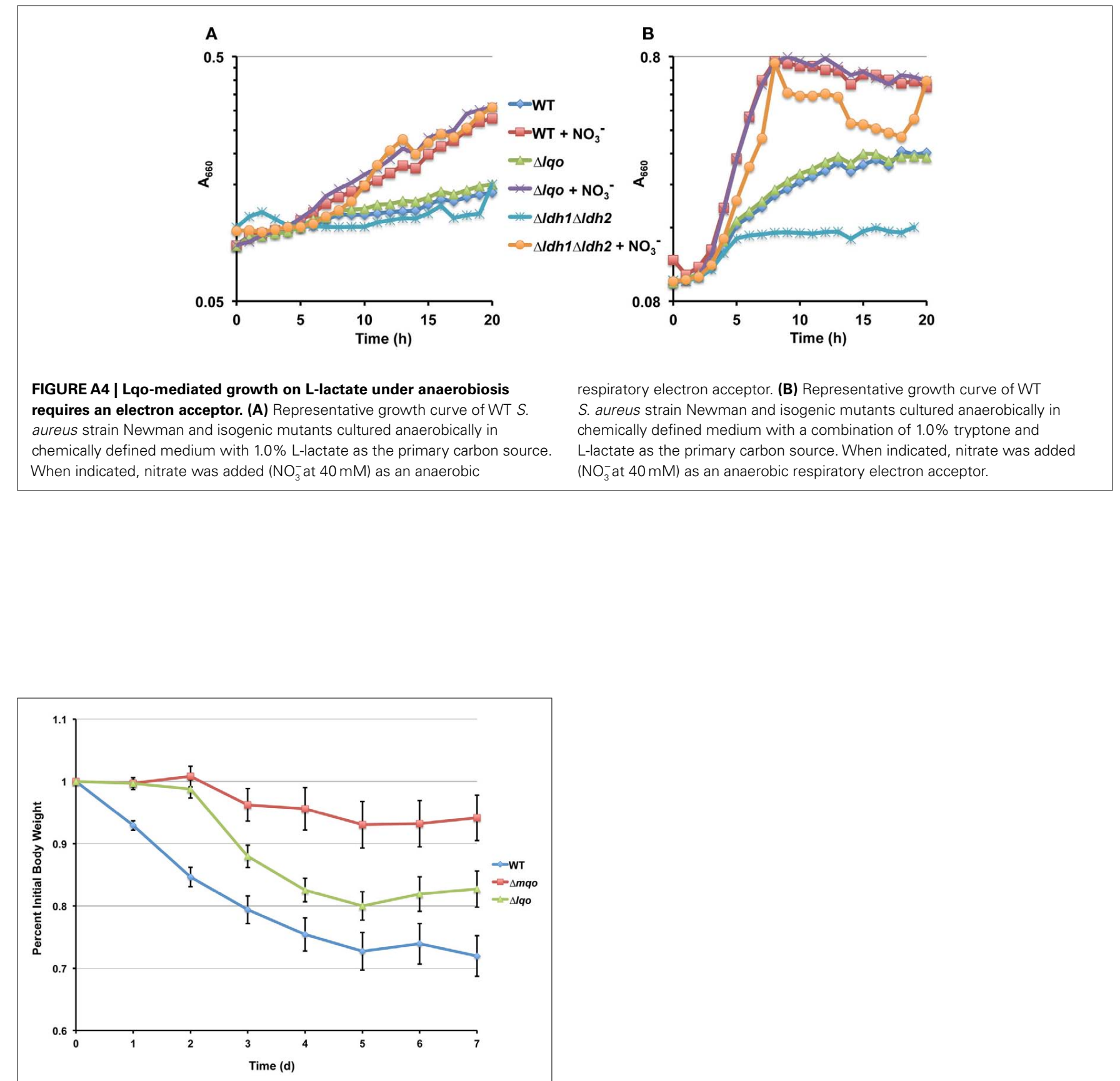

FIGURE A5 | Both Mqo and Lqo are required for full virulence in

S. aureus. Eight female C57BL/6 mice (two independent experiments each involving four mice per group) were inoculated i.v. with $5 \times 10^{6} \mathrm{cfu}$ of $S$. aureus strain Newman or isogenic mutants. Weight loss was documented and mice losing $\geq 30 \%$ of their body weight at the time of inoculation were sacrificed as per approved IACUC protocols. 


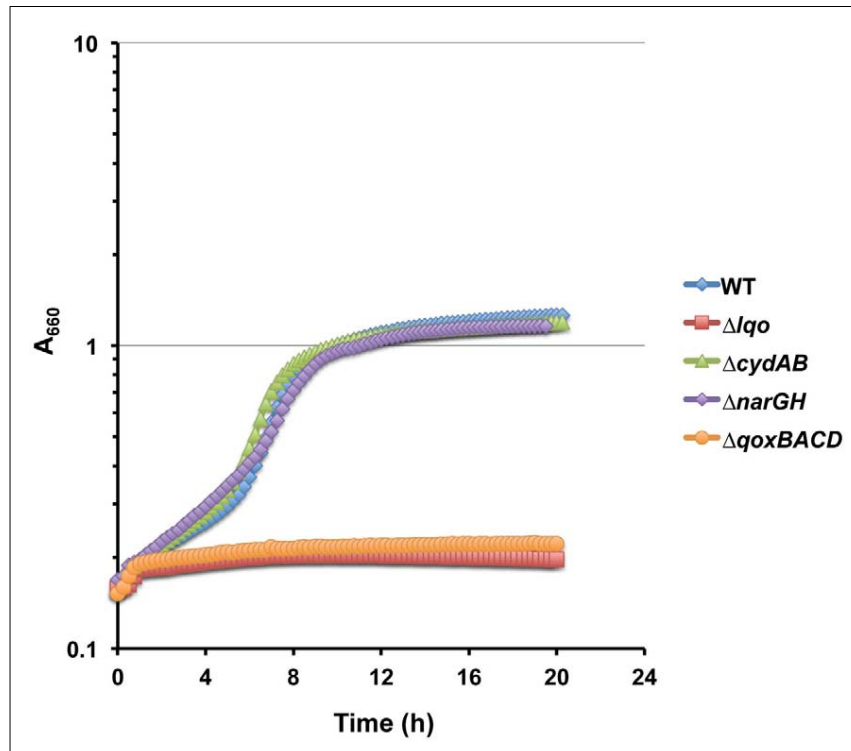

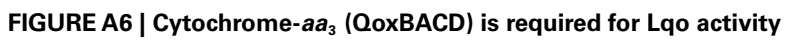
during NO.-stress. Representative growth curve on WT S. aureus strain $\mathrm{COL}$ and isogenic mutants grown in chemically defined medium using a combination of $0.1 \%$ L-lactate $/ 1.0 \%$ tryptone as primary carbon sources in the presence of low level NO. (administered as $10 \mathrm{mM}$ DETA/NO when cultures reach $\mathrm{A}_{660}=0.15$ ). 